Vanda Maria da Rosa Jardim ${ }^{1}$

Luciane Prado Kantorski ${ }^{1}$

Michele Mandagará de Oliveira ${ }^{1}$

Carlos Alberto dos Santos Treichel ${ }^{1}$

Cândida Garcia Sinott Silveira Rodrigues ${ }^{1}$

Leticia Valente Dias ${ }^{1}$
${ }^{1}$ Departamento de Enfermagem, Faculdade de Enfermagem e Obstetrícia, Universidade Federal de Pelotas. R. Gomes Carneiro 1, Porto Pelotas. 96010610 Pelotas RS Brasil. vandamrjardim@gmail.com

\section{Limitações de comportamento social entre usuários da Rede de Atenção Psicossocial no sul do Brasil}

\author{
Limitations of social behavior among users \\ of the psychosocial care network in the south of Brazil
}

Abstract This cross-sectional study aims to assess through analysis of data suggested by the Social Behavior Schedule (SBS) quantitatively and qualitatively distinguishing between the limitations of social interaction presented by users of substitute services. Analyses were performed of 385 instruments applied to users of Therapeutic Residential Services (SRT) and the Centers for Psychosocial Care (CAPS). Twenty-one items from the Social Behaviour Schedule (SBS, BR). The data indicate that the individuals studied had from zero to 15 social behavior problems, with an average of 2.5 problems per service user with a standard deviation of 2.84. The majority of the sample appeared to have 1-2 problems among those identified by the scale, and the nonspecific behaviors are present in greater proportion in both services, followed by panic attacks among CAPS users and problems with personal appearance and hygiene in SRT users. In this way, the use of tools that make it possible to assess the data on the social aspects of the psychological distress deserves to be encouraged as a way to further research in this area, and rethink ways of effecting social reintegration of these individuals.

Key words Health evaluation, Community mental health services, Social behavior, Social behavior limitations, SBS
Resumo Este estudo transversal objetiva aferir, por meio da análise dos dados sugeridos pela escala SBS distinguindo de forma quantitativa e qualitativa, as limitações de convívio social apresentadas por usuários de serviços substitutivos. Foram analisados 385 instrumentos aplicados a usuários de Serviços Residenciais Terapêuticos (SRT) e de Centros de Atenção Psicossocial (CAPS). Foram utilizados os 21 itens da Social Behaviour Schedule (SBS-BR). Os dados nos indicam que os indivíduos estudados apresentavam de nenhum a 15 problemas de comportamento social entre os identificados pela escala. Apresentando uma média de 2,5 problemas por usuário com um desvio padrão de 2,84. A maior parte da amostra pareceu apresentar de 1 a 2 problemas entre os passiveis de serem identificados pela escala, estando os comportamentos inespecíficos em maior proporção em ambos serviços, seguido de ataques de pânico em usuários de CAPS e problemas com aparência pessoal e de higiene em usuários de SRT. Nesse sentido, o uso de instrumentos que nos permitam aferir os dados a cerca dos aspectos sociais do indivíduo em sofrimento psíquico merece ser estimulado como forma de continuar a pesquisa nessa área, $e$ repensar modos de efetivar a reinserção social desses sujeitos.

Palavras-chave Avaliação em saúde, Serviços comunitários de saúde mental, Comportamento social, Limitações de comportamento social, SBS 


\section{Introdução}

A partir dos anos 90 o processo de reorientação das ações em saúde mental com desinstitucionalização de pessoas com longo histórico de internação tornou-se política pública no Brasil e ganhou grande impulso com uma série de normatizações do Ministério da Saúde, oriundas do ano de 2002 que previam mecanismos para a redução de leitos psiquiátricos ${ }^{1}$.

A partir dessa nova política nacional de saúde mental, baseada na desinstitucionalização, a atenção ao portador de transtorno mental prestada em instituições fechadas deslocou-se para espaços extra-hospitalares, capazes de valorizar a manutenção dos vínculos sociais e familiares dos indivíduos ${ }^{2}$.

Nesta perspectiva, destacam-se na reforma psiquiátrica brasileira os Centros de Atenção Psicossocial (CAPS), serviço comunitário aberto do Sistema Único de Saúde regulamentado pelas portarias MS 336/02 $2^{3}$ e $2841 / 10^{4}$ que visam oferecer cuidados clínicos e de reabilitação psicossocial aos indivíduos com transtorno mental dentro de seu território ${ }^{5}$. E também os Serviços Residenciais Terapêuticos (SRT), ou Residências Terapêuticas regulamentadas pela portaria MS $106 / 2000^{6}$, casas localizadas no espaço urbano que atendem a necessidade de moradia de pessoas com histórico de longa internação que não possuem ou que tiveram seus vínculos familiares rompidos ${ }^{7}$. Entre as potencialidades desses serviços está o favorecimento da ressocialização do usuário, uma vez que possibilita ao mesmo estar socialmente inserido e dotado de papéis e funções sociais oriundos da vida em sociedade.

No entanto, projetos que apontam para ressocialização do indivíduo em sofrimento psíquico sofrem críticas no país e fora dele. Alguns autores $^{8,9}$ apontam a existência de um grupo de indivíduos que apresenta dificuldade na reinserção à sociedade, sugerindo inclusive sua inaptidão desses sujeitos para tratamento em serviços comunitários de saúde mental. Em geral, trata-se de indivíduos que passaram por longos períodos de internação e desenvolveram uma série de limitações de convívio social.

Avaliar o desempenho social de usuários dos serviços comunitários no contexto da política de saúde mental no Brasil surge então como uma necessidade a fim de diagnosticar e intervir em possíveis fatores limitantes para reabilitação psicossocial do sujeito. Assim como conhecer as associações entre as limitações e o perfil dos indivíduos.
Entre as escalas que têm sido utilizadas para medir as dificuldades no comportamento social de pacientes psiquiátricos, destaca-se a Social Behaviour Schedule (SBS). Que tem se mostrado um instrumento adequado, principalmente por ser tanto uma das mais utilizadas, favorecendo assim a comparação, como por sua capacidade em avaliar as pessoas mais comprometidas, que têm dificuldades para responder às questões propostas, cujas informações são fornecidas por um informante que conhece bem o indivíduo ${ }^{10}$.

Estudos anteriores têm trabalhado com a escala em populações de serviços comunitários de saúde mental em outras localidades do país, encontrando diferentes resultados. Por exemplo, estudo que encontrou uma proporção de indivíduos sem problemas correspondente a $16,5 \%{ }^{11}$ como outro em que essa parcela correspondia a $36,9 \%{ }^{12}$ da amostra. Essa comparação dos resultados entre diferentes regiões, serviços e momentos estimula uma ampla discussão quanto aos aspectos estudados.

Nessa perspectiva, esse trabalho visa aferir as limitações de comportamento social em usuários de SRT e CAPS no estado do Rio Grande do Sul, levando em conta variáveis sociodemográficas, além de dados quanto ao tempo de permanência no serviço e histórico de internação.

\section{Metodologia}

Trata-se de um estudo transversal, recorte da análise quantitativa dos instrumentos aplicados a usuários de Serviços Residenciais Terapêuticos (SRT) e de Centros de Atenção Psicossocial (CAPS) de cinco municípios do estado do Rio Grande do Sul (Alegrete; Bagé; Caxias do Sul; Porto Alegre; Viamão) entre os meses de setembro e dezembro de 2009. Os dados utilizados integram a Pesquisa intitulada "Redes que reabilitam - avaliando experiências inovadoras de composição de redes de atenção psicossocial (Redesul)" realizada a partir de abordagem quantitativa e qualitativa.

A amostra é de 385 usuários e compreende indivíduos de todos os 33 Serviços Residenciais Terapêuticos cadastrados no estado do Rio Grande do Sul até o período de estudo. Foram incluídos na amostra usuários de CAPS intensivo ou semi-intensivo de 6 CAPS dos municípios estudados. Do total da amostra, 20 usuários utilizavam concomitantemente os dois serviços.

O projeto possui aprovação pelo comitê de Ética em Pesquisa da Faculdade de Odontologia 
da Universidade Federal de Pelotas, com parecer favorável ao seu desenvolvimento, o qual se deu conforme o preconizado pela resolução CNS n ${ }^{\circ}$ $196 / 96^{13}$ e atende a resolução CNS no $466 / 12^{14}$ que regulamenta as pesquisas envolvendo Seres Humanos.

A fim de avaliar as limitações no comportamento social dos usuários dos serviços, foi realizada a aplicação da Social Behaviour Schedule (SBS-BR), escala composta por 21 itens com escores de 0 a 4 . A escala conta com duas formas de pontuação, o método BSS (Severe Behaviour Problems Scores), cujo problema é ausente quando a pontuação é 0 , considerado leve quando a pontuação das respostas é 1 ou 2 e considerado severo quando a pontuação é 3 ou 4 . E o método de pontuação BSM (Mild and Severe Behaviour Problems Score), escolhido para esse estudo. Neste, escores 0 e 1 indicam a ausência do problema e 2, 3 e 4 sugerem sua presença. A soma do total dos quesitos positivos fornece o BSM total de cada sujeito da amostra e a média da soma dos pontos de todos os sujeitos fornece o BSM global (BSM overall) da população estudada.

Elaborada por Wykes e Sturt ${ }^{15}$, a Social Behaviour Schedule avalia as limitações do comportamento social de pacientes de longa permanência, tanto em hospitais psiquiátricos quanto em serviços residenciais na comunidade. Sua validação para o Brasil foi realizada por Lima et al..$^{10} \mathrm{e}$, assim como em sua versão original, conta com escores variando de zero (ausência de problema) a quatro (problema severo).

Foram utilizadas variáveis sociodemográficas relacionadas a fim de identificar a distribuição das limitações de comportamento social conforme sexo (Masculino; Feminino), estado civil (Solteiro; Casado; Separado/Divorciado/Viúvo) e escolaridade (Sem escolaridade; Ensino fundamental; Ensino médio; Ensino superior) além de dados quanto ao tempo de permanência no serviço e histórico de internação.

Sua construção, processamento e análise dos dados deram-se por meio dos Softwares EPI INFO e STATA ${ }^{16}$, com dupla digitação e limpeza do banco de dados por digitadores independentes. O controle de qualidade contou com a replicação de $10 \%$ das entrevistas realizadas. Dada a distribuição normal das variáveis, o valor de $\mathrm{p}$ foi calculada por chi $^{2}$ de Pearson. Significância estatística foi definida como $\mathrm{p}<0,05$.

\section{Resultados}

Os dados apresentados na Tabela 1 nos indicam que entre os problemas de comportamento social identificados pela escala na amostra total, os comportamentos inespecíficos foram citados com maior frequência, de forma que sua proporção corresponde a 25,79\% dos indivíduos estudados. Entre os demais problemas que se destacam encontram-se os de inatividade, ataques de pânico/fobias e aparência pessoal/higiene.

Os problemas entre a amostra total encontrados em menores proporções ficam a cargo respectivamente das ideias ou comportamentos suicida ou de autoagressão $(3,41 \%)$, comportamento sexual inadequado $(3,65 \%)$, realização de ideias bizarras $(4,68 \%)$, comportamento destrutivo $(4,97 \%)$ e interação social no que se refere à habilidade de fazer contados sociais de forma adequada (7,35\%).

Ao analisar a população desse estudo de acordo com seu serviço de origem, os dados na Tabela 1 demonstram que há diferença quanto aos problemas mais prevalentes entre usuários de CAPS e SRT. Observa-se que há uma diferença estatística significativa nos itens: Conversação incoerente $(\mathrm{p}=0.04)$; Conversação excêntrica/inapropriada $(\mathrm{p}=0.00)$; Aparência pessoal/higiene $(\mathrm{p}=0.00)$; Inatividade $(\mathrm{p}=0.03)$; Problemas não especifica$\operatorname{dos}(\mathrm{p}=0.002)$.

Contudo, no que se refere aos problemas sociais de menor frequência, as populações de CAPS e SRT reproduzem resultados semelhantes, de forma que nos dois serviços esses problemas são observados em baixa proporção entre os indivíduos estudados.

Os dados do estudo nos indicam ainda que os usuários de serviços de saúde mental estudados apresentam de nenhum a 15 problemas de comportamento social entre os identificados pela escala SBS, apresentando uma média de 2,5 problemas por usuário do serviço com um desvio padrão de 2,84 .

A Tabela 2 expõe a quantidade de limitações sociais identificados por pessoa na população estudada, relacionada com dados considerados relevantes para comparação como: sexo, estado civil, escolaridade, tempo de permanência no serviço e histórico de internação. Através de sua análise é possível discernir que embora uma parcela considerável de indivíduos não apresente nenhum dos problemas de comportamento social citado, $32,73 \%$ dos usuários de serviços de saúde mental estudados apresenta de 1 a 2 fatores limitantes passíveis de serem reconhecidos pela escala. 


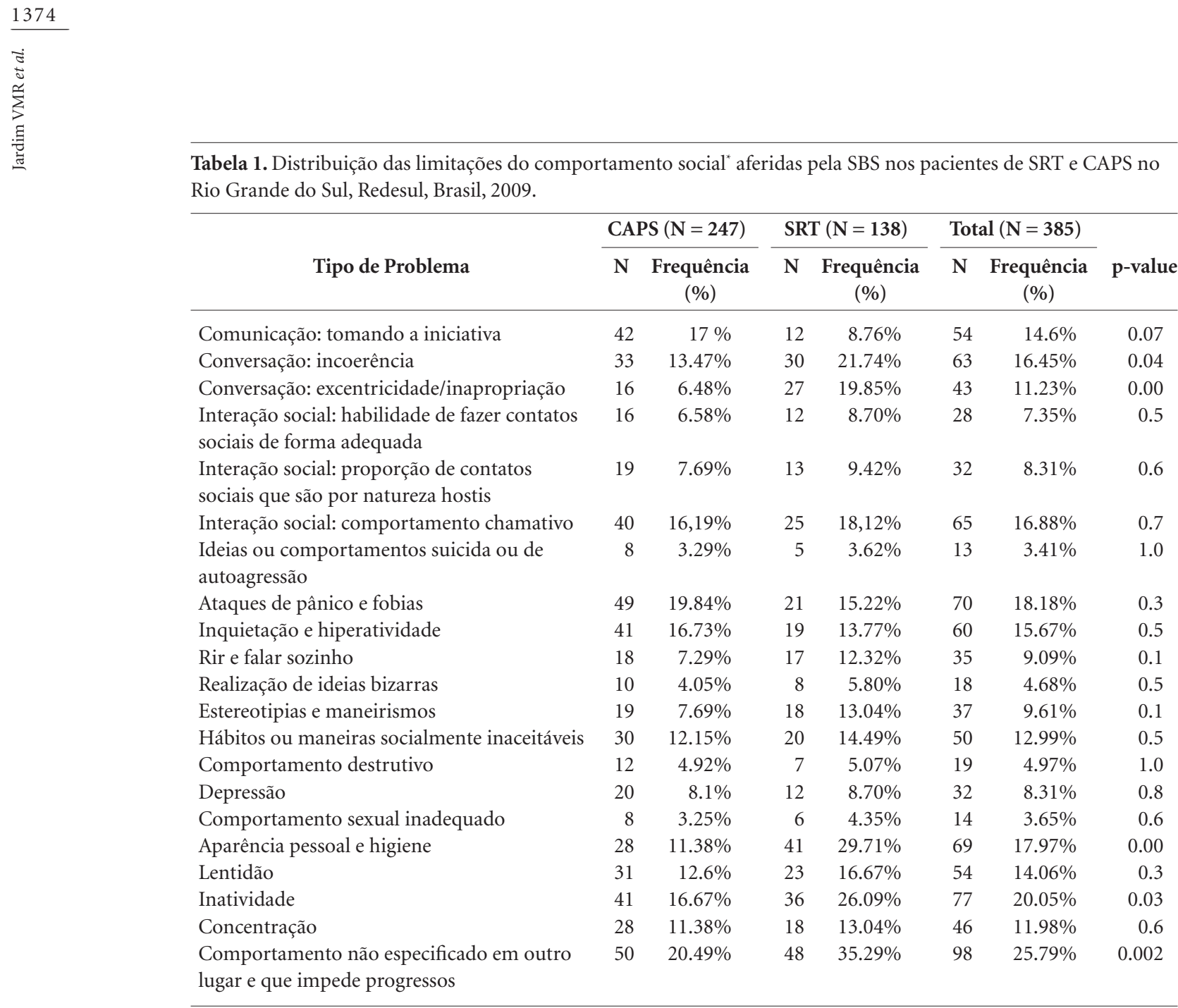

*Pontuação 2 ou mais nos 21 itens da SBS. Fonte: Redesul, 2009.

Todavia também é importante considerar a existência de uma parcela da população que apresenta um número acentuado de problemas (6 ou mais) sendo essa correspondente à 12,47\% dos indivíduos.

Quanto aos fatores sociodemográficos selecionados, bem como aos dados relacionados ao tempo de permanência no serviço, a Tabela 2 nos indica que homens apresentaram proporção maior de problemas. Entre a população estudada a proporção de mulheres sem nenhum problema foi de $36,26 \%$, enquanto de homens foi $22,17 \%$. No mesmo sentido, quando se observam os demais estratos, os homens sempre apresentam uma proporção maior de problema que as mulheres.

Ainda é possível identificar que indivíduos casados apresentaram menor proporção $(3,70 \%)$ de número acentuado de problemas (6 ou mais). Por outro lado, usuários separados/divorciados/ viúvos são os que proporcionalmente respondem pelo maior número de indivíduos sem nenhum problema $(41,07 \%)$. Indivíduos sem escolaridade que não apresentaram problemas representam apenas 13,75\% dos mesmos, já 17,50\% destes apresentaram 6 ou mais problemas.

Possuir alguma escolaridade parece ser fator de proteção quanto aos problemas analisados na população estudada. Percebe-se na Tabela 2 que entre indivíduos sem escolaridade, os que não apresentaram problemas são minoria. Porém entre os demais estratos de problemas, esses indivíduos figuram em maior proporção que os demais sujeitos, exceto no estrato de 3-5 problemas.

Entre os indivíduos que não possuem histórico de internação psiquiátrica, 52,17\% não apresentaram nenhum dos problemas identificados pela escala, enquanto entre os indivíduos que já foram internados, a proporção de indivíduos que não apresentou nenhum problema foi de $21,65 \%$. 
Tabela 2. Frequência de problemas apresentados por indivíduo entre os usuários de SRT e CAPS no Rio Grande do Sul de acordo com suas especificidades, Brasil, 2009.

\begin{tabular}{|c|c|c|c|c|c|c|c|c|}
\hline & \multicolumn{8}{|c|}{ Quantia de problemas } \\
\hline & \multicolumn{2}{|c|}{ Nenhum } & \multicolumn{2}{|c|}{$1-3$} & \multicolumn{2}{|c|}{ 3-5 } & \multicolumn{2}{|c|}{$6 \mathrm{ou}+$} \\
\hline & $\mathrm{N}$ & $(\%)$ & $\mathbf{N}$ & $(\%)$ & $\mathrm{N}$ & $\%$ & $\mathbf{N}$ & $\%$ \\
\hline \multicolumn{9}{|l|}{ Sexo } \\
\hline Mulheres & 66 & $36,26 \%$ & 57 & $31,32 \%$ & 43 & $23,63 \%$ & 16 & $8,79 \%$ \\
\hline Homens & 45 & $22,17 \%$ & 69 & $33,99 \%$ & 57 & $28,07 \%$ & 32 & $15,76 \%$ \\
\hline \multicolumn{9}{|l|}{ Estado civil } \\
\hline Solteiro & 59 & $23,79 \%$ & 87 & $35,08 \%$ & 65 & $26,21 \%$ & 37 & $14,92 \%$ \\
\hline Casado & 29 & $35,80 \%$ & 28 & $34,57 \%$ & 21 & $25,93 \%$ & 3 & $3,70 \%$ \\
\hline Separado/divorciado/viúvo & 23 & $41,07 \%$ & 11 & $19,64 \%$ & 14 & $25 \%$ & 8 & $14,29 \%$ \\
\hline \multicolumn{9}{|l|}{ Escolaridade ${ }^{*}$} \\
\hline Sem escolaridade & 11 & $13,75 \%$ & 35 & $43,75 \%$ & 20 & $25 \%$ & 14 & $17,50 \%$ \\
\hline Ensino fundamental & 63 & $31,50 \%$ & 60 & $30 \%$ & 52 & $26 \%$ & 25 & $12,50 \%$ \\
\hline Ensino médio & 30 & $38,46 \%$ & 22 & $28,21 \%$ & 20 & $25,64 \%$ & 6 & $7,69 \%$ \\
\hline Ensino superior & 5 & $29,41 \%$ & 6 & $35,29 \%$ & 4 & $23,53 \%$ & 2 & $11,76 \%$ \\
\hline \multicolumn{9}{|l|}{ Tempo de permanência no SRT } \\
\hline Até 1 ano & 8 & $27,59 \%$ & 8 & $27,59 \%$ & 9 & $31,02 \%$ & 4 & $13,79 \%$ \\
\hline $13-60$ meses & 5 & $12,82 \%$ & 15 & $38,46 \%$ & 14 & $35,90 \%$ & 5 & $12,82 \%$ \\
\hline 61-ou + meses & 5 & $25 \%$ & 8 & $40 \%$ & 4 & $20 \%$ & 3 & $15 \%$ \\
\hline Não respondeu/Não sabe & 3 & $6 \%$ & 21 & $42 \%$ & 18 & $36 \%$ & 8 & $16 \%$ \\
\hline \multicolumn{9}{|l|}{ Tempo de permanência no CAPS } \\
\hline Até 1 ano & 26 & $37,14 \%$ & 22 & $31,43 \%$ & 17 & $24,29 \%$ & 5 & $7,14 \%$ \\
\hline $13-60$ meses & 42 & $36,52 \%$ & 34 & $29,57 \%$ & 27 & $23,48 \%$ & 12 & $10,43 \%$ \\
\hline $61-\mathrm{ou}+$ meses & 19 & $39,58 \%$ & 14 & $29,17 \%$ & 7 & $14,58 \%$ & 8 & $16,67 \%$ \\
\hline Não Respondeu/Não sabe & 8 & $23,53 \%$ & 10 & $29,41 \%$ & 10 & $29,41 \%$ & 6 & $17,65 \%$ \\
\hline \multicolumn{9}{|l|}{ Histórico de interação } \\
\hline Não & 48 & $52,17 \%$ & 25 & $27,17 \%$ & 11 & $11,96 \%$ & 8 & $8,70 \%$ \\
\hline Sim & 63 & $21,65 \%$ & 100 & $34,36 \%$ & 88 & $30,24 \%$ & 40 & $13,75 \%$ \\
\hline Amostra total & 111 & $28,83 \%$ & 126 & $32,73 \%$ & 100 & $25,97 \%$ & 48 & $12,47 \%$ \\
\hline
\end{tabular}

*Houveram perdas, amostra estudada $\mathrm{n}=375 .{ }^{* *}$ Houveram perdas, amostra estudada $\mathrm{n}=383$. Fonte: Redesul, 2009

\section{Discussão}

O comportamento do indivíduo em sofrimento psíquico historicamente foi utilizado como justificativa para sua exclusão social ${ }^{17}$. Nesse sentido, é importante aferir as habilidades sociais do indivíduo, a fim de diagnosticar a existência de fatores limitantes para sua ressocialização, e intervir nos aspectos identificados como dificultadores.

Porém, é importante refletir quanto ao que seria de fato um comportamento social inadequado por parte do portador de um sofrimento psíquico, uma vez que em comunidade se exige um padrão de comportamento no qual tudo o que foge do que é "correto e bom" é tido como um fator que dificulta a convivência, assim como se pode observar nos discursos de familiares de usuários de CAPS em estudo já realizado. O portador de transtornos mentais é considerado aquele que não age como as pessoas normais, que não se comporta de acordo com normas sociais e familiares, que não se expressa e pensa adequadamente ${ }^{18}$.

Estimular a reflexão quanto a essa perspectiva é uma necessidade constante, visto que entre os indivíduos desse estudo, por exemplo, 28,83\% não apresentaram nenhum problema de comportamento social, assim como $36,9 \%$ dos participantes de estudo anterior, realizado por Gonçalves et al. ${ }^{12}$. Os achados desse estudo ${ }^{18}$ mostram ainda que embora a maioria dos indivíduos apresente algum problema de comportamento social, a maior parte deles os apresenta em baixa quantidade, já que 32,73\% dos sujeitos estudados apresentaram apenas 1 ou 2 problemas dos 21 identificados pela escala.

O mesmo acontece com estudo de Gonçalves et al. ${ }^{12}$, no qual $28,3 \%$ da amostra apresentou a mesma condição. 
Alguns estudos encontraram resultados que sugerem indivíduos mais comprometidos. No estudo de Abelha et al. ${ }^{11}$, a proporção de indivíduos sem problemas foi de $16,5 \%$ e a maior concentração de indivíduos $(34,8 \%)$ foi na faixa de 3 a 5 problemas. O referido estudo encontrou ainda uma parcela de $26,1 \%$ de indivíduos que apresentou mais de 5 problemas entre os identificados pela escala. Nesse estudo, a parcela de indivíduos que apresentaram 6 ou mais problemas foi de $12,47 \%$.

Como sugerido em outros estudos ${ }^{8,9}$, esses dados remetem a existência de um grupo de indivíduos que possuem mais limitantes sociais que outros. Porém, não são suficientes para indicar a inaptidão desses sujeitos para tratamento em serviços comunitários de saúde mental, já que ao analisar a prevalência de cada problema, assim como em outros estudos prévios, verificou-se baixa prevalência de limitações em áreas que pudessem representar riscos ao indivíduo e à sociedade, tais como comportamento suicida ou autoagressão, comportamento sexual inadequado e destrutividade.

$\mathrm{Na}$ amostra total desse estudo, a prevalência foi de $3,41 \%$; 3,65\%; e 4,97\% para comportamento suicida ou autoagressão, comportamento sexual inadequado e destrutividade respectivamente. Não houve diferença estatística significante entre as médias encontradas nos CAPS e nos SRT. O que sugere que mesmo indivíduos advindos de longos históricos de internação, e que em tese seriam mais comprometidos, apresentam baixo risco a si e aos demais.

Por outro lado, houve itens na escala com diferença estatística entre as médias apresentadas nos usuários de cada serviço, sugerindo diferenças quanto à proporção desse tipo de problemas entre os mesmos. As diferenças se deram nos seguintes problemas: Comportamento não especificado em outro lugar e que impede progressos $(p=0,002)$; Conversação: incoerência $(p=0,04)$; Conversação: excentricidade/inapropriação ( $p$ $=0,00)$; Aparência pessoal e higiene $(p=0,00)$; Inatividade $(p=0,03)$. Estes itens figuram entre os mais prevalentes na população estudada e, em todos estes, a prevalência foi proporcionalmente maior entre indivíduos atendidos em SRT.

É importante ressaltar que indivíduos que passaram por históricos de internação parecem apresentar mais problemas que os outros. Os dados apresentados na Tabela 2 deste estudo corroboram com essa perspectiva. Já que enquanto $52,17 \%$ dos indivíduos que não passaram por internações não apresentaram nenhum problema de comportamento social, essa proporção cai para $21,65 \%$ entre os indivíduos que já foram internados. É possível perceber ainda que entre indivíduos que apresentam numero mais acentuado de problemas, como no caso de 3 a 5 problemas, há uma notória diferença entre as proporções.

Nesta mesma perspectiva, os resultados obtidos em estudo de 2007 realizado em Barbacena $(\mathrm{MG})^{19}$ indicam efeitos significativos relacionados ao histórico de internação. Seus autores puderam observar uma relação inversamente proporcional entre o tempo de internação e o escore de evolução, em que quanto menor o tempo de internação maior o escore de evolução ${ }^{19}$.

Tanto as diferenças estatísticas entre itens importantes por sua prevalência na população estudada, como o maior número de problemas entre indivíduos que passaram por internações, convergem para uma reflexão de o quanto os problemas de comportamento social listados são características natas desse sujeito ou são sequelas de sua história pregressa, introjetadas pela lógica de cuidado asilar. Já que em isolamento o sujeito não era estimulado a exercitar sua capacidade de relacionamento e a desenvolver sua autonomia, tendo sua interação social restringida.

Os resultados deste estudo induzem ainda a reflexão quanto ao problema mais prevalente entre a população estudada. O problema de convívio social não especificado teve prevalência de 20,49\% entre indivíduos atendidos no CAPS e $35,29 \%$ entre indivíduos de SRT.

Esse item se refere a um comportamento ou atitude não abordados nos demais itens da escala que parecem impedir o progresso do indivíduo, pode compreender distúrbios do sono; distúrbios alimentares; anorexia; tabagismo; roubo e obsessões. O alto índice dessa limitação na população estudada sugere a diversidade de comportamentos considerados prejudiciais em sociedade. Estes podem estar relacionados com as particularidades e subjetividades do sujeito. Portanto, ressalta-se a importância de primar por um processo de reabilitação psicossocial individualizado e coerente com as demandas de cada indivíduo, estimulando o sujeito de acordo com suas necessidades.

É complexo prever o que resulta em maiores dificuldades no relacionamento em comunidade do indivíduo em sofrimento psíquico. Se a quantidade de problemas que o individuo possui ou se a ligação com a forma com que o problema é visto pelo grupo social ao qual pertence como mais grave ou mais aceitável. É pertinente observar que estas considerações podem variar de acor- 
do com aspectos culturais e históricos do núcleo em que o individuo se encontra. Portanto, mais que entender o indivíduo em transtorno mental como um portador de problemas e dificuldades na sua vida social, é necessário considerar suas potencialidades e desenvolve-las em conjunto. De forma que sua inserção social seja efetiva.

Nesse sentido, a rede substitutiva em saúde mental, na qual se inserem CAPS e SRT, parece ser uma importante aliada no contexto da reforma psiquiátrica brasileira em favor da reabi- litação psicossocial do indivíduo em sofrimento psíquico. Já que busca diminuir o dano causado pelo transtorno mental, envolvendo família e comunidade, estendendo assim o círculo social do usuário ${ }^{20}$, favorecendo o estímulo ao desenvolvimento de sua capacidade de socialização.

Este estudo apresenta como limitação o período da coleta dos dados, realizada no ano de 2009, porém, seus dados estimulam a continuação de pesquisas nessa área possibilitando comparações futuras.

\section{Colaboradores}

VMR Jardim, LP Kantorski, MM Oliveira, CAS Treichel, CGSS Rodrigues e LV Dias participaram igualmente de todas as etapas de elaboração do artigo. 


\section{Referências}

1. Alverga AR, Dimenstein M. A reforma psiquiátrica e os desafios na desinstitucionalização. Interface (Botucatu) 2006; 10(20):299-316.

2. Estevam MC, Marcon SS, Antonio MM, Munari DB, Waidman MAP. Convivendo com transtorno mental: perspectiva de familiares sobre atenção básica. Rev Esc Enferm USP 2011; 45(3):677-684.

3. Brasil. Ministério da Saúde (MS). Portaria n. ${ }^{\circ} 336$ GM, de 19 de fevereiro de 2002. Diário Oficial da União 2002; 20 fev.

4. Brasil. Ministério da Saúde (MS). Portaria No 2.841/ GM/MS, de 20 de setembro de 2010. Institui, no âmbito do Sistema Único de Saúde - SUS, o Centro de Atenção Psicossocial de Álcool e outras Drogas - 24 horas - CAPS AD III. Diário Oficial da União 2010; 21 set.

5. Ramminger T, Brito JC. "Cada CAPS é um CAPS": Uma coanálise dos recursos, meios e normas presentes nas atividades dos Trabalhadores de Saúde mental. Psicologia \& Sociedade 2011; 23(esp):150-160.

6. Brasil. Ministério da Saúde (MS). Portaria No 106, de 11 de fevereiro de 2000. Institui os Serviços Residenciais Terapêuticos. Diário Oficial da União 2000; 12 fev.

7. Brasil. Ministério da Saúde (MS). Secretaria de Atenção à Saúde. Departamento de Ações Programáticas Estratégicas. Residências terapêuticas: o que são, para que servem. Brasília: MS; 2004.

8. Trieman N, Leff J. Long-termoutcome of long-stay psychiatric in-patients considered unsuitable to live in the community. Br J Psychiatry 2002; 181:428-432

9. Trieman N, Gooch C. The TAPS Project 33: A prospective follow-up study of long-stay patients discharged fromtwo psychiatric hospitals. Am J Psychiatry 1996, 153(10):1318-1324

10. Lima LA, Gonçalves S, Lovisi G, Pereira BB. Validação transcultural da Escala de Avaliação de Limitações no Comportamento Social - SBS-BR. Rev. psiquiatr. clín. 2003; 30(4):126-138.

11. Abelha L, Muñoz MD, Gonçalves S, Fagundes P, Barbosa DR, Legay LF, Lovisi G. Evaluation of social disablement, psychiatric symptoms and autonomy in long-stay psychiatric patients. Rev. psiquiatr. Clin. 2006; 33(1):10-17.
12. Gonçalves S, Fagundes P, Lovisi G, Lima LA. Avaliação das limitações no comportamento social em pacientes psiquiátricos de longa permanência. Cien Saude Colet 2001; 6(1):105-113.

13. Brasil. Ministério da Saúde (MS). Conselho Nacional de Saúde. Resolução no 196 de 10 de outubro de 1996. Diretrizes e Normas Regulamentadoras de Pesquisas Envolvendo Seres Humanos. Diário Oficial da União 1996; 16 out.

14. Brasil. Ministério da Saúde (MS). Conselho Nacional de Saúde. Resolução no 466, de 12 de dezembro de 2012. Diário Oficial da União 2013; 13 jun.

15. Wykes T, Sturt E. The measurement of social behaviou in psychiatric patients: an assessment of the reliability and validity of the SBS schedule. Br J Psychiatry 1986, 148:1-11.

16. StataCorp. Stata Statistical Software: Release 11. College Station: StataCorp LP; 2009.

17. Spadini LS, Souza MCB. A doença mental sob o olhar de pacientes e familiares. Rev. Esc. Enfer. USP 2006; 40(1):123-127.

18. Colvero LA, Ide CAC, Rolim MA. Família e doença mental: a difícil convivência com a diferença. Rev. Esc. Enferm. USP 2004; 38(2):197-205.

19. Vidal CEL, Gontijo ECDM, Bandeira MB. Avaliação das habilidades de vida independente e comportamento social de pacientes psiquiátricos desospitalizados. Rev. psiquiatr. Rio Gd. Sul 2007; 29(3):294-304.

20. Mielke FB, Kantorski LP, Olschowsky A, Jardim VMR. Características do cuidado em saúde mental em um CAPS na perspectiva dos profissionais. Rev. Trab. educ. saúde 2011; 9(2):265-276.

Artigo apresentado em 16/06/2014

Aprovado em 18/10/2014

Versão final apresentada em 20/10/2014 